\title{
BIOMETRIA DE UNA POBLACION DE HOMALASPIS PLANA (MILNE EDWARDS, 1834) EN PUNTA MAULE (CORONEL, CHILE)
}

\author{
MARCO A. RETAMAL Y RODOLFO QUINTANA
}

Instituto de Biología, Universidad de Concepción, Chile.

\section{SYNOPSIS}

This work deals with the bionomy of the population of Homalaspis plana (Milne Edwards, 1834) sampled in Coronel, Chile. Homalaspis plana share his biotope with: Paraxanthus barbiger (Poeppig, 1836) and Gaudichaudia gaudichaudi (Milne Edwards, 1834). A marked sexual dimorphism is apparent, the males are larger and heavier than the females; moreover, the volumes of the chelae of the first are also larger. The percentaje of the female population was significantly larger than the males population. The spawning extends from June to De. cember. Each class of the ovigerous females, gives a different contribution, the maxima is done by the $5,74 \mathrm{~cm}$ class with $29,21 \%$. The mini. mum size of ovigerous females is $49,3 \mathrm{~mm}$ L.C.

In: roduccion

Los recursos renovables del Mar Chileno son poco conocidos, entre ellos se encuentran los crustáceos decápodos, cuyas especies expiotadas alcanzan a 20, destacándose los "langostinos" del Centro y Sur de Chile, la "centolla" del Sur, y las "langostas" de la región insular. En este último tiempo los brachyura han empezado a tomar gran auge con su industrialización y exportación y siendo su biología prácticamente desconocida, ha llevado a los investigadores chilenos a produndizar sus estudios en estos tópicos, siendo este trabajo una pequeña contribución en el logro de estos propósitos.

\section{Sistematica}

Homalaspis plana (Milne Edwards, 1834) Rathbun, M. 1930 , p. 288-289, láminas 128-130.

Este Brachyura pertenece a la familia Xanthidae, que agrupa a 24 especies de la costa chilena. Los caracteres diagnósticos para este género monoespecífico se pueden resumir: "Superficie cubierta de gránulos gruesos y deprimidos. Margen anterior de la frente bilobulado, separado por una fisura en forma de "V". La frente que es inclinada nace más allá del pedúnculo antenal. Quelípodos macizos con dentes fuertes. Dactilos de las patas ambulatorias con manchones de pelos suaves y muy cortos, a modo de tapiz, cubriendo gran parte de la articulación" (Fig. 1).

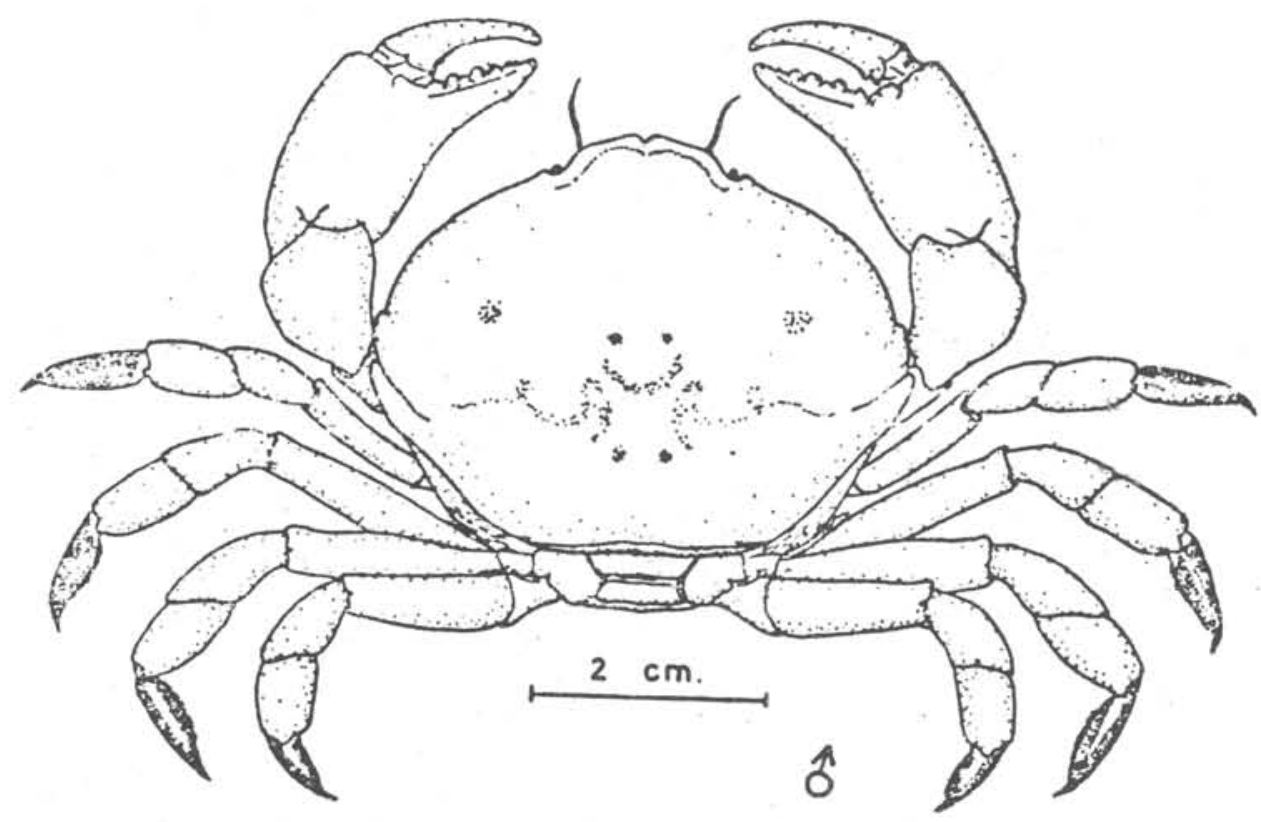

Fig. 1-Homalaspis plana.

\section{Distribucion Geografica y Batimetrica}

Su distribución geográfica es muy amplia, extendiéndose desde Guayaquil (Ecuador) hasta el Estrecho de Magallanes (Chile). También se encuentra en el Archipiélago de Juan Fernández (fide Garth. 1957).

Su rango batimétrico varía entre 0 y $18 \mathrm{~m}$ (Antezana et al., $1965: 43)$.

Para nuestro país es un recurso comercial importante entre los braquiuros. y el área de mayor extracción comercial es el Golfo de Arauco.

\section{Materiales y Metodos}

Los ejemplares de Homalaspis plana fueron obtenidos de la zona sublitoral en Punta Roca Maule, Coronel, Golfo de Arauco (37001' $45^{\prime \prime}$; $\left.73^{\circ} 10^{\prime} 55^{\prime \prime} \mathrm{W}\right)$ (F ig. 2).

\section{Muestreo y Tratamiento de Las Muestras}

Los especímenes analizados provenían de las capturas que realizan los pescadores artesanales. 


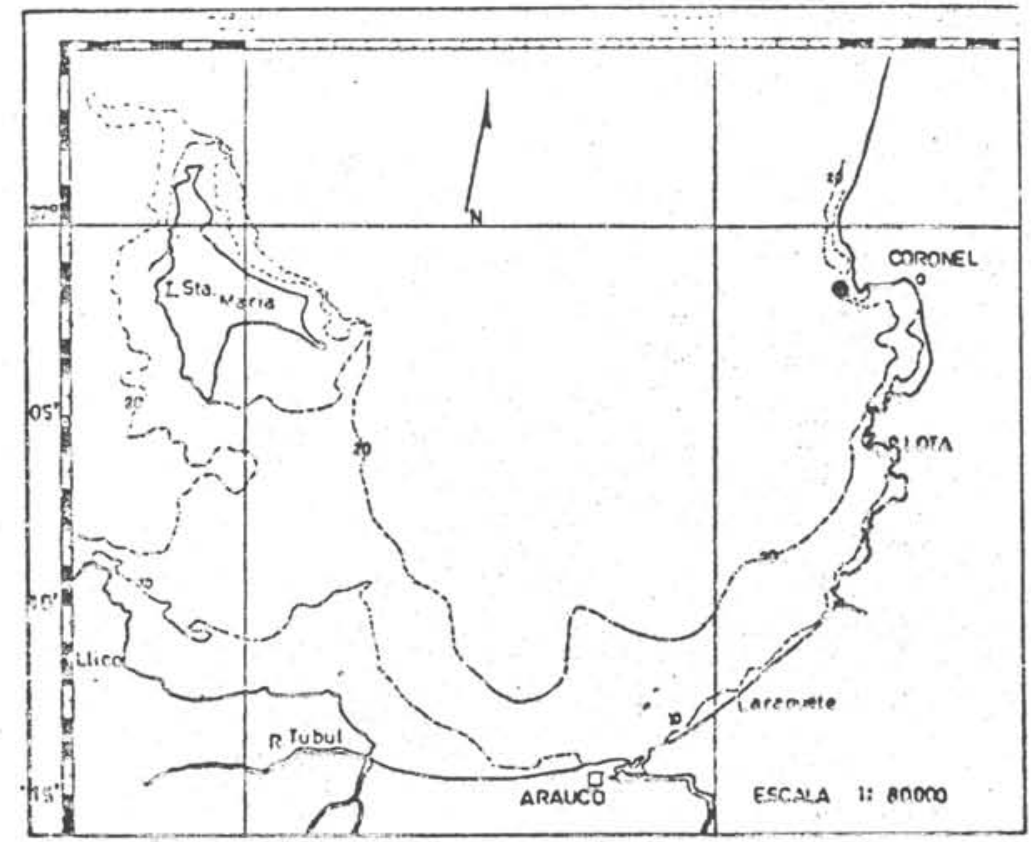

Fig. 2-Zona de muestreo, Golfo de Arauco.

El presente estudio parcial de la población de esta especie connrennde un periodo de observaciones mensuales çue abarcó desde aoril de 1970 a mayo de 1971 , en el cual se analizó un total de 1.407 ejemplares. Mensualmente se obtenía alrededor de 100 individuos.

Las observaciones no merísticas efectuadas son:

- Determinación de sexo

- Presencia de huevos y numero de ellos en hembras ovígeras

- Identificación de los estados de las ovas

- Presencia de epibiontes.

Las observaciones meristicas son:

- Longitud máxima del cefalotórax (L.C.)

- Ancho máximo del cefalotórax (A.C.)

- Peso húmedo vs Longitud cefalotorácica

- Diametro de los huevos

Para obtener la fecundidad se calculó el número de huevos presentes en 30 hembras. Los valores obtenidos se promediaron y pulieron según el método de Karlovac (1953) usado por Bahamonde (1958) (Fig. 5).

Se calculó también las relaciones entre peso húmedo y longitud cefalotorácica para ambos sexos, obteniéndose una relación positiva. Además, el peso promedio fue ploteado con los meses para cada uno de los sexos.

\section{Observaciones exologicas}

Los especímenes analizados sólo corresponden a la población adulta proveniente del sublitoral, ya que los juveniles se encuentran en la zona mesolitoral. La especie en estudio vive en sustratos rocosos junto a Paraxanthus borbiger (Poeppig, 1836) y Gaudichaudia gaudichaudi (Milne Edwards, 1834) ambos de la familia Xanthidae. Otro organismo de la fauna acompañante es Ophiophragmus chilensis (Muller y Troschel, 1843) Ophiuroidea. En los apéndices torácicos se encontró gran cantidad de Hirudíneos, así como también Cirrripedios sobre el caparazón y pereiopodos.

\section{Sexualidad y Reproduccion}

Las diferencias sexuales se presentan externamente en la forma del abdomen y en la ubicación de las aberturas genitales.

Durante el período de muestreo el porcentaje de hembras es superior al de los machos, generalmente muy por sobre el $50 \%$, excepto en septiembre y octubre, meses en los cuales estos valores fueron de 46 y $49 \%$, respectivamente (Fig. 3 ).

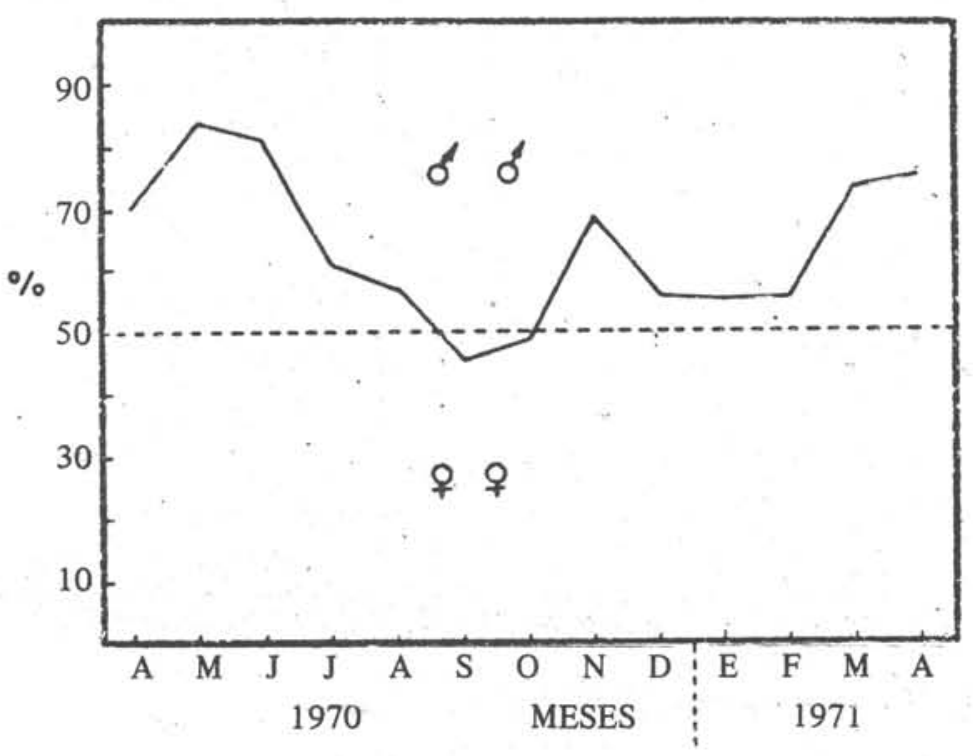

Fig. 3-Proporción de sexos. 
El período de desove observado se extiende desde junio a diciembre. Durante este lapso se pudo observar huevos en estado
I; en cambio, los huevos en estado II aparecen sólo entre septiembre y diciembre (Fig. 4).

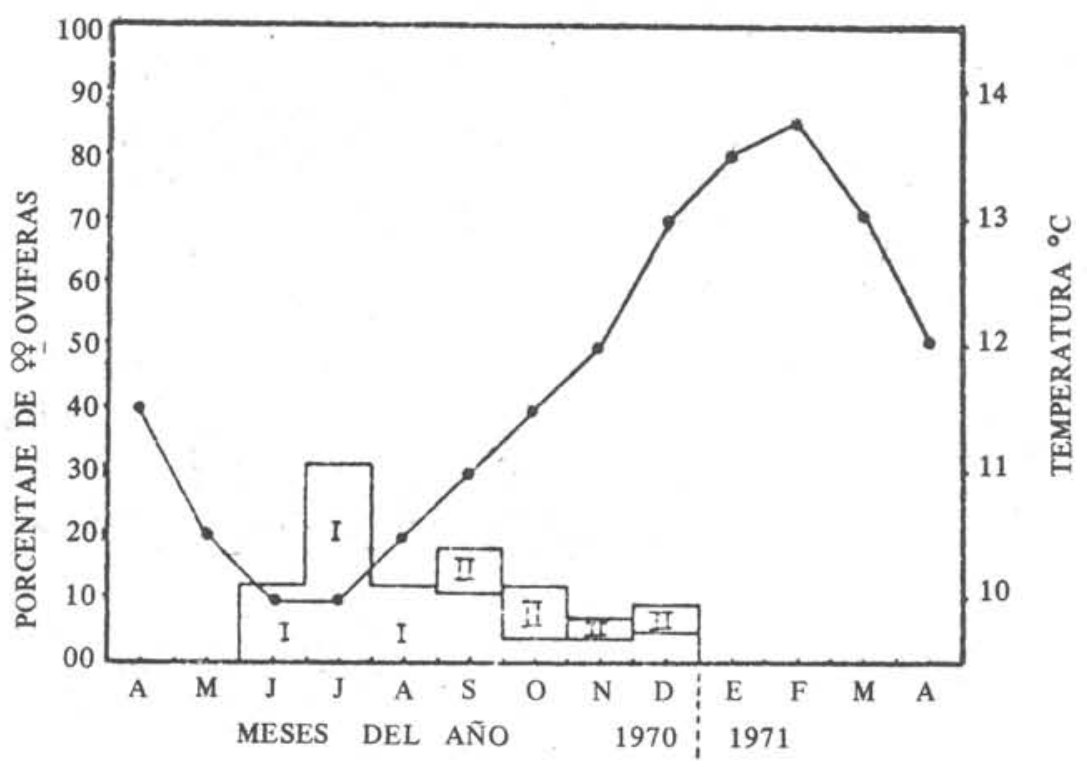

Fig. 4-Período de desove y su relación con la temperatura.

Los huevos al estado 1 presentan una coloración rojıza homogénea, en cambio en el estado Il su color es violáceo; con respecto al diámetro para el primer estado oscila entre 460 y 540 $\mu$ y entre 600 y $660 \mu$ para el segundo estado.

La fecundidad se calculó sobre la base del número de huevos que portaban 30 hembras. Con las maximas y minimas absolutas se obtuvo un promedio pulido que entregó como valor mínimo 153.659 y como máximo 342.566 huevos, mostrando el gráfico correspondiente una clara correlación positiva entre el número de huevos y la talla de las hembras (Fig. 5).

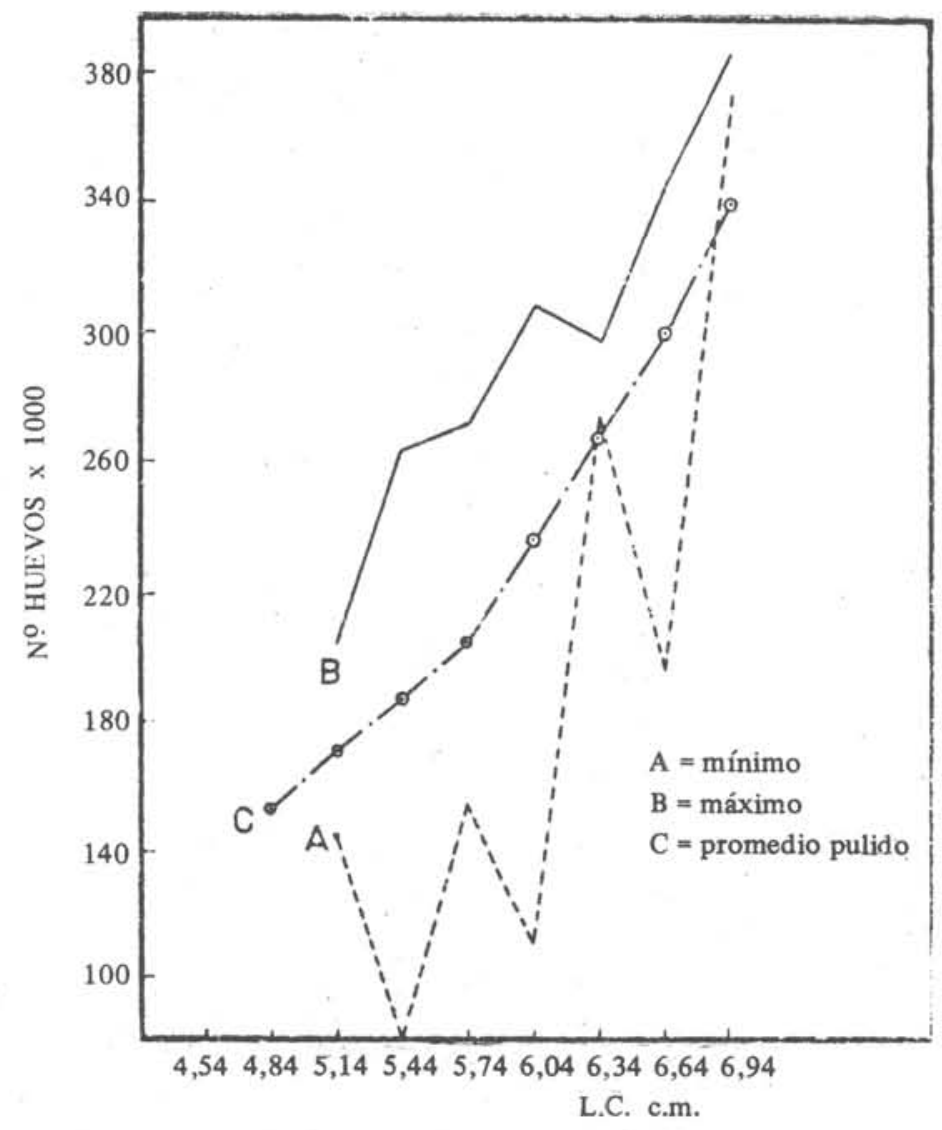

Fig. 5-Longitud del cefalotórax/número de huevos en hembras de Homalaspis plana.

Como se demuestra claramente en la Figura 6, los mayores aporte de crías lo hacen las clases intermedias no por su alta fecundidad, sino por su mayor frecuencia en la población.
Expresado en porcentajes acumulativos, este aporte de crías alcanza el $65,42 \%$ si consideramos solamente 3 clases de tamaño que son las más abundantes en la población 


\section{Discusion y Conclusiones}

Homalaspis plana habita en la zona muestreada junto a otro dos Xanthidae: Paraxanthus barbiger y Gaudichaudia gaudichaudi. Sus epibiontes son hirudíneos y cirripedios que se localizan sobre los apéndices locomotores y el cefalotórax.

Durante el período de muestreo se observó una gran dominancia de hembras muy por sobre el $50 \%$, alcanzando incluso $85,86 \%$ en mayo. Los machos sobrepasan el $50 \%$ sólo en 2 meses.

El período de desove abarca desde junio a diciembre.

Comparando la curva de fecundidad obtenida por Antezana et al. (op. cit.) y la obtenida con nuestros resultados, podemos concluir que se amplía el rango de talla mínima para las hembras ovígeras de $55.0 \mathrm{~mm}$ a $49.3 \mathrm{~mm}$

La fecundidad está en relación directa con la longitud cefalotorácica (L.C.). Así, un especimen de $48.4 \mathrm{~mm}$ porta 142.766 huevos y uno de $69.4 \mathrm{~mm}$ un total de 379.073 huevos como promedio, siendo el valor máximo observado de 387.536 huevos.

El diámetro de los huevos en estado I varía entre 460 y 540 $\mu$ y entre 000 y $690 \mu$ para nuevos en estado $u$.

El mayor aporre de crias a la población lo realizan las clases intermedias, no por su alta fecundidad, sino por su alta frecuencia; es por lo tanto aconsejable, por parte de los organismos pertinentes, la protección de estas tallas durante la época de desove, de manera de proteger el recurso.

\section{Referencias bibliográficas}

ANTEZANA, T.;FAGETTI, E. \& LOPEZ, M.T. 1965. Observaciones bio-ecológicas en decápodos comunes en Valparaíso. Revta Biol. mar., 12(1/3) : 1-60.

BAHAMONDE, M. 1958. Sobre la fecundidad de la gamba o camarón nailon (Heterocarpus reedi Bahamonde). Investnes zool. chil., $4: 259-264$.

GARTH, J.S. 1957. The Crustacea Decapoda Brachyura of Chile. Reports of the Lund University Chile Expedition 1948-49. Lunds Univ. Arsskrift, N.F., Avd. 2, 53 (7) : 1-130.

KARLOVAC, O. 1953. An ecological study of Nephrops norve. gicus (L.) of the High Adriatic. Rep. Inst. oceanogr. Split, $5(2 \mathrm{C}): 1-51$.

RATHBUN, M.J. 1930. The cancroid crabs of America of the families Euryalidae, Portunidae, Atelecyclidae, Cancridae and Xanthidae. Bull. U.S. natn. Mus., $152: 1-593$. 